\title{
Prevalence of fibromyalgia in fourteen Korean tertiary care university hospital pain clinics
}

This article was published in the following Dove Press journal: Journal of Pain Research

\author{
Ho-Jin Lee' \\ Eunjoo Choi' \\ Francis Sahngun Nahm' \\ Seong-Soo $\mathrm{Choi}^{2}$ \\ Young-Hoon $\mathrm{Kim}^{3}$ \\ Jee Youn Moon ${ }^{4}$ \\ Shin Hyung Kim ${ }^{5}$ \\ Chan-Woo Cho ${ }^{6}$ \\ Pyung Bok Lee ${ }^{1,7}$ \\ 'Department of Anesthesiology \\ and Pain Medicine, Seoul National \\ University Bundang Hospital, \\ Seongnam, Republic of Korea; \\ ${ }^{2}$ Department of Anesthesiology and \\ Pain Medicine, Asan Medical Center, \\ Seoul, Republic of Korea; ${ }^{3}$ Department \\ of Anesthesiology and Pain Medicine, \\ Seoul St. Mary's Hospital, Seoul, \\ Republic of Korea; ${ }^{4}$ Department of \\ Anesthesiology and Pain Medicine, \\ Seoul National University Hospital, \\ Seoul, Republic of Korea; ${ }^{5}$ Department \\ of Anesthesiology and Pain Medicine, \\ Severance Hospital, Seoul, Republic of \\ Korea; ${ }^{6}$ Seoul Chan Pain Clinic, Seoul, \\ Republic of Korea; ${ }^{7}$ Department of \\ Anesthesiology and Pain Medicine, \\ Seoul National University, Seoul, \\ Republic of Korea
}

Correspondence: Pyung Bok Lee Department of Anesthesiology and Pain Medicine, Seoul National University

Bundang Hospital, Gumi-dong 300,

Bundang-gu, Seongnam-si I3620, Republic of Korea

Email painfree@snubh.org
Purpose: We aimed to investigate the prevalence of fibromyalgia (FM) in patients who visited outpatient pain clinics in Korea, using the modified 2010 American College of Rheumatology (ACR) criteria and to evaluate comorbid conditions that may be associated with FM.

Patients and methods: Outpatients, aged $\geq 18$ years, who first visited pain clinics at any of the 14 tertiary care university hospitals, were recruited for this study. Modified 2010 ACR criteria were used to diagnose FM. Participants' demographic information and eleven comorbid measures (sleep disturbance, fatigue, irritable bowel syndrome, amnesia, hypothyroidism, headache, anxiety, depression, rheumatism, low back pain, and history of traffic accident) were also investigated via additional questionnaires.

Results: We recruited 1,233 outpatients (754 men and 479 women). Based on the modified 2010 ACR criteria, $13.38 \%$ (165/1,233 patients, 95\% CI: $11.48-15.28)$ of patients had FM. As participants with a history of FM were excluded, the prevalence of FM was $11.0 \%(123 / 1,118$ patients). Patients with FM were more likely to have one or more of the eleven predetermined comorbid conditions. Patients who were female and who had a previous history of FM, sleep disturbance, headache, or a history of traffic accident were more likely to have FM.

Conclusion: FM, according to the modified 2010 ACR criteria, appears to be a common disorder among chronic pain patients referred to tertiary care university hospital pain clinics. Therefore, physicians treating pain should consider this prevalence of FM among pain clinic patients.

Keywords: tertiary care centers, chronic pain, diagnosis, fibromyalgia

\section{Introduction}

Fibromyalgia (FM) is a condition characterized by chronic soft tissue pain with a number of comorbidities. ${ }^{1}$ Epidemiological studies have reported that the prevalence of FM is $\sim 2 \%-4 \%$ in the general population and is much more common in women than in men, as well as among the elderly. ${ }^{2}$ FM is typically associated with chronic widespread pain and tenderness to palpation, resulting from a generalized low pain threshold. Currently, this change in threshold is attributed to changes in various neurotransmitters in patients with a genetic predisposition to $\mathrm{FM}^{2}$

The first uniform diagnostic criteria for FM were developed by the American College of Rheumatology (ACR) in 1990. These 1990 ACR criteria required the presence of at least 11 of 18 specified tender anatomical points upon palpation. Although "palpation" is defined as the application of about $4 \mathrm{~kg}$ of force, in clinical practice this procedure may vary depending on the physician. Therefore, this criterion has been criticized as subjective and time-consuming. ${ }^{1}$ The 1990 ACR criteria also failed to 
account for many typical comorbid symptoms experienced by most FM patients. Due to these shortcomings, in 2010, new ACR criteria (2010 ACR criteria), with an emphasis on easy application in clinical practice, were proposed. ${ }^{3}$ In these revised 2010 criteria, a widespread pain index (WPI), a specific type of self-report scale, was included in place of the previous tender point diagnostic criteria. A symptom severity (SS) scale was also proposed, with a focus on the comorbid symptoms experienced by FM patients.

Despite improvements, the 2010 ACR criteria, as the 1990 criteria before them, also required a physician to assess the presence and severity of comorbid symptoms. In 2011, diagnostic criteria (the modified ACR 2010 criteria) were developed, which were the partially modified 2010 diagnostic criteria without the requirement for physician assessment of somatic symptoms. ${ }^{4}$ The modified ACR 2010 criteria could thus be implemented more easily and at a lower cost. ${ }^{1}$ The physician assessment of somatic symptoms was replaced with a summary score representative of three, self-reported symptoms. By using these modified ACR 2010 criteria in primary care, physicians were able to diagnose FM with adequately high sensitivity and specificity. ${ }^{5}$

Despite the extensive application and use of these modified ACR criteria elsewhere, 5,6 their use in assessing the prevalence of FM in Korea has not yet been investigated. There are only two epidemiological studies of FM in Korea, both of which are based on the 1990 ACR criteria. ${ }^{7,8}$ A further study compares the fit of 1990 and 2010 ACR criteria in 98 patients already diagnosed with FM. ${ }^{9}$ In addition, most previous studies have been conducted in patients recruited from a general population or recruited directly from rheumatology clinics. No studies have specifically assessed patients at multicenter pain clinics. Recently, it has been reported that FM presents as a continuous spectrum, rather than as a single disease form, and is often accompanied by various other chronic pain disorders. ${ }^{6,10}$ For these reasons, we targeted patients with chronic pain who visited tertiary care university hospital pain clinics for our assessments of FM in the present study. In addition, we employed the modified ACR 2010 criteria, which can be employed without a physician and thus are potentially more useful than the 1990 diagnostic criteria, which required significant physician's time and effort.

The aim of this study was to determine the prevalence of FM in patients who visited outpatient pain clinics in Korea using the modified 2010 ACR criteria and to examine them for comorbid conditions that may be associated with FM.

\section{Patients and methods}

\section{Patients}

This is a cross-sectional questionnaire survey-based study. Each hospital's institutional review board approved the study (Asan Medical Center, Chonnam National University, Chungnam National University, Daejeon St. Mary’s Hospital, Jeju National University School of Medicine, Korea University Guro Hospital, National Cancer Center, Pusan National University, Samsung Medical Center, Seoul National University Bundang Hospital, Seoul National University Hospital, Seoul St. Mary’s Hospital, Severance Hospital, and Wonkwang University Hospital). We recruited outpatients, aged $\geq 18$ years, who first visited pain clinics from 14 tertiary care university hospitals. Written informed consent was obtained from all participants. Patients who did not agree to participate or who had difficulty understanding the questionnaires were excluded.

\section{Modified 2010 ACR criteria}

We used the modified 2010 ACR criteria to evaluate FM (Table 1). As the Korean version of these modified 2010 ACR criteria has not yet been validated, a three-step translation process was applied to the original version. First, two anesthesiologists translated the modified 2010 ACR criteria into Korean. Second, one bilingual person, who had completed high school in the US, translated the Korean version back into English. Third, two native English speakers compared the original questionnaire with the reverse-translated questionnaire and assessed each item for its degree of concordance using a 7-point scale, with one point indicating no concordance and seven points indicating perfect concordance. Any items that were scored between 5 and 7 points were adopted, while items that did not meet these criteria were subjected to a review process. The five people involved in the translation process are not included as authors for this article. The Korean translation of the modified 2010 ACR criteria used in this study is available in the Supplementary material.

All the participants completed the modified 2010 ACR criteria questionnaire prior to meeting with a pain physician. According to the 2010 ACR criteria, this questionnaire consisted of two scales: the WPI and the SS scale. Furthermore, according to the modified 2010 ACR criteria, the requirement for a physician's estimate of the extent of somatic symptoms was eliminated and substituted with a sum score based on three specific, self-reported symptoms (headaches, pain or cramps in lower abdomen, and depression). ${ }^{4}$ In addition, a new fibromyalgia symptom (FS) scale, based on the sum of 
Table I Fibromyalgia criteria modified from ACR diagnostic criteria

Criteria:

A patient satisfies the modified ACR 2010 fibromyalgia diagnostic criteria if the following three conditions are met: (I) Widespread pain index $\geq 7$ and symptom severity score $\geq 5$ or widespread pain index between 3 and 6 and symptom severity score $\geq 9$; (2) symptoms have been present at a similar level for at least 3 months; and (3) the patient does not have a disorder that would otherwise sufficiently explain the pain.

Ascertainment:

I) Widespread pain index: Note the number of areas in which the patient has had pain over the last week. In how many areas has the patient had pain? Score will be between 0 and 19.

Shoulder girdle, Lt. Hip (buttock, trochanter), Lt. Jaw, Lt. Upper Back

Shoulder girdle, Rt. Hip (buttock, trochanter), Rt. Jaw, Rt. Lower Back

Upper Arm, Lt. Upper Leg, Lt. Chest, Neck

Upper Arm, Rt. Upper Leg, Rt. Abdomen

Lower Arm, Lt. Lower Leg, Lt.

Lower Arm, Rt. Lower Leg, Rt.

2) Symptom severity score: fatigue, waking unrefreshed, and cognitive symptoms.

For the each of these three symptoms, indicate the level of severity over the past week using the following scale:

$0=$ no problem; I = slight or mild problems: generally mild or intermittent; 2 = moderate: considerable problems, often present and/or at a moderate level; 3 = severe: pervasive, continuous, life-disturbing problems.

The symptom severity score is the sum of the severity of the three symptoms (fatigue, waking unrefreshed, and cognitive symptoms) plus the sum of the number of the following symptoms occurring during the previous 6 months: headaches, pain or cramps in lower abdomen, and depression (0-3). The final score is between 0 and 12 .

Note: Adapted from Wolfe et al. J Rheumatol. 201 I;38(6): I I 3-1 I22, with permission of The Journal of Rheumatology. ${ }^{4}$

Abbreviations: ACR, American College of Rheumatology; Lt, left; Rt, right.

the WPI and SS scales, is proposed in the modified 2010 criteria. ${ }^{4} \mathrm{FS}$ scale scores range from 0 to 31 , with 13 points or more serving as a cutoff point for FM diagnosis. It has been reported that an FS scale score of $\geq 13$, based on the 1990 criteria, correctly classified $93 \%$ of patients and had a specificity of $96.6 \%$ and a sensitivity of $91.8 \%{ }^{4}$ In the present study, we also investigated the prevalence of FM using FS scale and the optimal FS scale cutoff value for the diagnosis of FM based on the modified 2010 ACR criteria. We also collected patient demographic information (sex and age) and assessed for the presence of 11 comorbid measures (insomnia, fatigue, irritable bowel syndrome, amnesia, hypothyroidism, headache, anxiety, depression, rheumatism, low back pain, and history of traffic accident) via additional questionnaires.

\section{Statistical analyses}

We used the "Sampsize" to calculate the number of subjects required for the study. ${ }^{11}$ According to the Korean Pain Society, the total number of patients who first visited tertiary care university hospital pain clinics participating in this study in 2015 was 26,900 . In a previous pilot work, we found that the prevalence of FM among individuals seen in tertiary care university hospital pain clinics was $\sim 25 \%$. The prevalence of rate in the previous pilot study was also obtained using the modified 2010 ACR criteria. Based on this result, we calculated the number of participants required for the present study to be 1,227 , accounting for a $10 \%$ dropout rate and with a $95 \%$ CI and an upper and lower error limit of $2.5 \%$. All statistical analyses were performed using $\mathrm{R}$ statistical software (version 3.2.3; R Foundation for Statistical Computing, Vienna, Austria). Data are presented as the mean \pm SD or as a number and percentage of total participants. Comparisons were made using the Student's $t$-test and Pearson's Chi-squared test. A receiver operating characteristic (ROC) analysis was used to determine the optimal FS score cutoff value for the diagnosis of FM according to the modified 2010 diagnostic criteria. The comorbid conditions associated with FM were examined using a multivariable logistic regression analysis. Independent variables with a $P$-value $<0.2$ in the simple univariate logistic regression analysis were further assessed by multiple logistic regression analysis. $P$-values $<0.05$ were considered statistically significant.

\section{Results}

From April 2016 to February 2017, a total of 1,233 patients (754 men and 479 women) from 14 tertiary care university hospital pain clinics completed the questionnaires. The prevalence of FM in this population according to the modified 2010 ACR criteria was $13.38 \%$ (165/1,233 patients, $95 \%$ CI: 11.48-15.28). Table 2 presents the prevalence of FM by sex and age. Because the prevalence of FM varies by age and sex, we calculated age- and sex-specific prevalence rates. The peak prevalence was observed in the fourth decade of life in women and the sixth decade of life in men. Among all the participants, 115 (35 men and 80 women) had previously 
Table 2 Prevalence of FM based on age and sex

\begin{tabular}{|c|c|c|c|c|c|c|c|}
\hline \multirow{2}{*}{$\begin{array}{l}\text { Age } \\
\text { (years) }\end{array}$} & \multicolumn{3}{|c|}{ Female } & \multicolumn{3}{|c|}{ Male } & \multirow[t]{2}{*}{ Total prevalence (\%) } \\
\hline & $\mathbf{N}$ & $\mathbf{F M}^{\mathbf{a}}$ & Prevalence (\%) & $\mathbf{N}$ & $\mathbf{F M}^{\mathbf{a}}$ & Prevalence (\%) & \\
\hline $20 \sim 29$ & 30 & 3 & 10 & 48 & 5 & 10.4 & 10.3 \\
\hline $30 \sim 39$ & 55 & 13 & 23.6 & 59 & 7 & 11.9 & 17.5 \\
\hline $40 \sim 49$ & 100 & 21 & 21 & 71 & 8 & 11.3 & 17 \\
\hline $50 \sim 59$ & 204 & 37 & 18.1 & 103 & 13 & 12.6 & 16.3 \\
\hline $60 \sim 69$ & 188 & 26 & 13.8 & 103 & 5 & 4.9 & 10.7 \\
\hline 70 79 & 130 & 20 & 15.4 & 74 & 2 & 2.7 & 10.8 \\
\hline $80 \sim$ & 47 & 5 & 10.6 & 21 & 0 & 0 & 7.4 \\
\hline Total & 754 & 125 & 16.6 & 479 & 40 & 8.4 & 13.4 \\
\hline
\end{tabular}

Note: a Diagnosed with FM.

Abbreviation: FM, fibromyalgia.

been diagnosed with FM. Of these, 42 participants met our classification criteria. As participants with a history of FM were excluded, the prevalence of FM was $11.0 \%$ (123/1,118 patients).

As suggested in a previous study, an FS score $\geq 13$, used as a cutoff value, allowed for the diagnosis of FM with high sensitivity and specificity. ${ }^{4}$ Using these criteria, the prevalence of FM in our cohort was 18.3\% (226/1,233; 63 men, 163 women). In addition, in our study, an FS score $\geq 12$ was found to be the optimal cutoff value for the detection of FM, as diagnosed according to the modified 2010 diagnostic criteria, with a sensitivity of $95.8 \%$ and a specificity of $88.6 \%$. The area under the ROC curves was 0.961 (95\% CI: 0.947-0.975; $P<0.001)$.

Table 3 summarizes co-occurrence rates of 11 comorbid measures (sleep disturbance, fatigue, irritable bowel syndrome, amnesia, hypothyroidism, headache, anxiety, depression, rheumatism, low back pain, and history of traffic accident) and FM diagnoses, according to the modified 2010 ACR diagnostic criteria. Patients with FM were more likely to have one or more of these 11 predetermined comorbidities.

Tables 4 and 5 show the results of simple and multiple logistic regression analyses in which the relationships between the presence of FM and demographic and comorbid conditions were analyzed. These analyses revealed that patients who were female and who had a previous history of FM, sleep disturbance, headache, or a history of traffic accident had significantly higher frequencies of FM compared with counterparts in each of these categories.

\section{Discussion}

This study serves as the first epidemiological study investigating the prevalence of FM in patients at pain clinics at multiple
Table 3 FM-related scores and associated factors in the FM group (as determined by the modified 2010 ACR criteria) and non-FM groups

\begin{tabular}{|c|c|c|c|}
\hline \multirow[t]{2}{*}{ Variable } & \multirow{2}{*}{$\begin{array}{l}\text { Non-FM group } \\
(N=1068)\end{array}$} & \multirow{2}{*}{$\frac{\text { FM group }}{(N=165)}$} & \multirow[t]{2}{*}{ Rate ratio } \\
\hline & & & \\
\hline WPI (0-19) & $3.0 \pm 2.1$ & $8.6 \pm 4.1$ & \\
\hline$S S(0-12)$ & $4.6 \pm 2.3$ & $7.9 \pm 2.0$ & \\
\hline $\mathrm{FS}(0-3 \mathrm{I})$ & $7.6 \pm 3.5$ & $16.5 \pm 4.5$ & \\
\hline Sex & & & $1.99(-)$ \\
\hline Male & 439 (4I.1\%) & $40(24.2 \%)$ & \\
\hline Female & $629(58.9 \%)$ & 125 (75.8\%) & \\
\hline Previous FMa & $73(6.8 \%)$ & $42(25.5 \%)$ & \\
\hline Fatigue & $24 I(22.6 \%)$ & $61(37.0 \%)$ & $\mathrm{I} .7(1.30-2.3 \mathrm{I})$ \\
\hline IBS & 154 (14.4\%) & $36(21.8 \%)$ & $1.6(1.14-2.24)$ \\
\hline Sleep disturbance & 435 (40.7\%) & $110(66.7 \%)$ & $1.7(1.28-2.34)$ \\
\hline Amnesia & $256(24.0 \%)$ & $73(44.2 \%)$ & $2.0(1.47-2.58)$ \\
\hline Hypothyroidism & $88(8.2 \%)$ & $26(15.8 \%)$ & $2.0(1.39-2.93)$ \\
\hline Headache & $146(13.7 \%)$ & 51 (30.9\%) & $2.4(1.78-3.20)$ \\
\hline Anxiety & 166 (15.5\%) & $46(27.9 \%)$ & $1.9(1.40-2.58)$ \\
\hline Depression & 131 (12.3\%) & $44(26.7 \%)$ & $2.3(1.69-3.21)$ \\
\hline Rheumatism & $91(8.5 \%)$ & $22(13.3 \%)$ & $1.7(1.10-2.48)$ \\
\hline LBP & $383(35.9 \%)$ & $85(51.5 \%)$ & $1.5(1.14-2.01)$ \\
\hline Traffic accident & 142 (I3.3\%) & 48 (29.1\%) & $2.3(1.72-3.12)$ \\
\hline
\end{tabular}

Note: aPreviously diagnosed with FM.

Abbreviations: ACR, American College of Rheumatology; FM, fibromyalgia; FS, fibromyalgia symptom scale; LBP, low back pain; SS, symptom severity scale; WPI, widespread pain index; IBS, irritable bowel syndrome.

tertiary care university hospitals. It is also the first study to employ the modified 2010 ACR diagnostic criteria in Korea.

Our results demonstrate that the prevalence of FM among the participants enrolled in our study was $13.38 \%$. Even if participants with a history of FM were excluded, the prevalence of FM was $11.0 \%$ (123/1,118 patients). This figure is higher than that identified in prior, broad epidemiological studies, only two of which have previously been conducted in Korea. ${ }^{7,8}$ One of these - a cohort study based on the assessment of FM in the general population $(n=1,028)$ - reported 
Table 4 Simple logistic regression analyses for factors associated with FM

\begin{tabular}{|c|c|c|c|c|}
\hline \multirow[t]{2}{*}{ Variables } & \multirow[t]{2}{*}{ OR } & \multicolumn{2}{|c|}{ Odds ratio $(95 \% \mathrm{Cl})$} & \multirow[t]{2}{*}{$P$-value } \\
\hline & & Lower & Upper & \\
\hline Age & 0.89 & 0.81 & 0.99 & 3.60 \\
\hline \multicolumn{5}{|l|}{ Sex } \\
\hline Male & 1.00 & & & \\
\hline Female & 2.18 & 1.51 & 3.21 & $<0.001$ \\
\hline Previous FMa & 4.65 & 3.03 & 7.08 & $<0.001$ \\
\hline Fatigue & 2.01 & $\mathrm{I} .42$ & 2.84 & $<0.001$ \\
\hline IBS & 1.66 & 1.09 & 2.47 & 0.02 \\
\hline Sleep disturbance & 2.91 & 2.07 & 4.14 & $<0.001$ \\
\hline Amnesia & 2.52 & 1.79 & 3.53 & $<0.001$ \\
\hline Hypothyroidism & 2.08 & 1.28 & 3.30 & $<0.001$ \\
\hline Headache & 2.83 & 1.93 & 4.09 & $<0.001$ \\
\hline Anxiety & 2.10 & $\mathrm{I} .43$ & 3.05 & $<0.001$ \\
\hline Depression & 2.60 & 1.75 & 3.82 & $<0.001$ \\
\hline Rheumatism & 1.65 & 0.98 & 2.67 & 0.05 \\
\hline LBP & 1.90 & 1.37 & 2.65 & $<0.001$ \\
\hline Traffic accident & 2.68 & 1.82 & 3.89 & $<0.001$ \\
\hline
\end{tabular}

Note: aPreviously diagnosed with FM.

Abbreviations: FM, fibromyalgia; IBS, irritable bowel syndrome; LBP, low back pain.

Table 5 Multiple logistic regression analyses for factors associated with FM

\begin{tabular}{|c|c|c|c|c|}
\hline \multirow[t]{2}{*}{ Variables } & \multirow[t]{2}{*}{ OR } & \multicolumn{2}{|c|}{ Odds ratio $(95 \% \mathrm{Cl})$} & \multirow[t]{2}{*}{$P$-value } \\
\hline & & Lower & Upper & \\
\hline \multicolumn{5}{|l|}{ Sex } \\
\hline Male & 1.00 & & & \\
\hline Female & 1.97 & 1.33 & 2.98 & $<0.001$ \\
\hline Previous FMa & 3.57 & 2.22 & 5.69 & $<0.001$ \\
\hline Fatigue & 0.95 & 0.63 & $\mathrm{I} .43$ & 0.81 \\
\hline IBS & 1.30 & 0.82 & 2.03 & 0.25 \\
\hline Sleep disturbance & 2.00 & 1.35 & 2.96 & $<0.001$ \\
\hline Amnesia & $\mathrm{I} .47$ & 0.99 & 2.17 & 0.06 \\
\hline Hypothyroidism & 1.32 & 0.77 & 2.21 & 0.30 \\
\hline Headache & 1.88 & 1.22 & 2.86 & $<0.001$ \\
\hline Anxiety & 0.86 & 0.48 & 1.51 & 0.61 \\
\hline Depression & 1.27 & $0.7 \mathrm{I}$ & 2.26 & 0.42 \\
\hline Rheumatism & 1.02 & 0.58 & 1.74 & 0.93 \\
\hline LBP & 1.34 & 0.93 & 1.92 & 0.11 \\
\hline Traffic accident & 1.89 & 1.23 & 2.87 & $<0.001$ \\
\hline
\end{tabular}

Note: aPreviously diagnosed with FM.

Abbreviations: FM, fibromyalgia; IBS, irritable bowel syndrome; LBP, low back pain.

a prevalence of 2.2\%. ${ }^{7}$ Another study included patients who visited a hospital primary care center and revealed an FM prevalence of $1.7 \%{ }^{8}$

There are two likely reasons for the difference between the prevalence found in these previous studies and that found in the present one. First, for example, one previous study used the 1990 ACR diagnostic criteria, ${ }^{12}$ while the present study used the modified 2010 diagnostic criteria. Because the modified 2010 ACR criteria are self-reported with no need for physician evaluation, resultant prevalence may be higher than that resulting from the use of the 1990 diagnostic criteria, which require the evaluation of patient tender points. In Scotland, the prevalence values of FM were reported to be $1.7 \%$ and $5.4 \%$ in the same population group according to the 1990 and modified 2010 ACR criteria, respectively. ${ }^{13}$ In the present study, a change in diagnostic sensitivity may have resulted in changed prevalence estimates, as determined by the modified ACR 2010 criteria.

A further reason for difference between the rates reported here and elsewhere may be differences in the participants included. While the participants included in previous studies were recruited from the general population or primary care center users, ${ }^{7,8}$ we recruited participants from tertiary care university hospital pain clinics within our hospital system. In Korea, the prevalence of FM has not previously been reported in the general population using the modified 2010 ACR criteria. Elsewhere, however, prevalence ranging from $0.6 \%$ to $6.4 \%$ has been reported according to the modified 2010 ACR criteria. ${ }^{6,14,15}$ A higher prevalence of pain symptoms in our study participants than that of the general population might additionally contribute to these differences in prevalence. The differences in the results of an epidemiological study performed in Scotland, which compared the two sets of criteria, suggest that the findings of this study may have reflected other factors besides differences in the classification criteria. ${ }^{13}$ We considered that the difference in the target patient group may have caused this difference. It is difficult to diagnose FM without clinical indications because it is a diagnosis of exclusion. In addition, as many patients with FM experience symptoms such as fatigue, sleep disturbance, mood swings, paralysis, and psychological instability, the diagnosis of FM tends to be delayed. ${ }^{16}$ Therefore, patients with FM are often referred to multiple specialists and undergo numerous clinical tests before a diagnosis is made. ${ }^{17}$ Choy et al reported that from the time patients recalled first presenting to a physician for their FM symptoms, it took an average of 2.3 years and consultation with 3.7 physicians before receiving a diagnosis. ${ }^{18}$ For this reason, tertiary care university hospital pain clinics patient populations likely have higher FM prevalence rates than those of the general population. Prior to our study, Brill et al reported that the prevalence of FM was $41.2 \%$ in patients who visited a tertiary care university hospital pain clinics, ${ }^{19}$ a much higher rate than that reported here. However, this rate was based on a small sample size ( 85 patients) and a single center, and the race of the patients studied was different. Thus, it is difficult to directly compare their results with 
those reported in the present study. We think that the results of our multicenter study, with a greater number of patients, should be more widely accepted.

A further result indicates that considerations of heterogeneity and demographic dynamics among clinical populations are valuable in understanding FM. In the present study, the prevalence of FM was found to be higher in women. The difference in FM prevalence between men and women was about $2: 1$ (16.6\% vs 8.4\%). In general, when using the 1990 ACR criteria including the tender point exam, the difference in FM prevalence between men and women was reported from 8:1 to $30: 1 .^{2}$ However, when using diagnostic criteria excluding the tender point test, such as the 2010 ACR criteria and the modified 2010 ACR criteria, the difference in FM prevalence between men and women is diminished from $4: 1$ to $1: 1 .^{2}$ In general, systemic symptoms such as fatigue and irritable bowel syndrome, which are commonly associated with FM, are known to occur more commonly in women, who are also known to have significantly more tender points. ${ }^{20}$ These variations lead to differences in prevalence according to sex. The mechanism of this difference is not known precisely, but it is known that various biological, psychological, and sociocultural factors will contribute. ${ }^{20}$

Results from the current study also revealed multiple additional factors, including sleep disturbance and headache, which were associated with FM. These results are consistent with previously published, known risk factors for FM in the general population. ${ }^{16,21,22}$ In addition, we identified a statistically significant relationship between participant's history of traffic accident(s) and the prevalence of FM in this study. Although little is known about the initiating cause(s) of FM, these may vary among subgroups of individuals, and it appears that physical trauma may be temporally related to the onset of symptoms in $>60 \%$ of cases. ${ }^{1}$ The review study by McLean et al suggested that traffic accidents can act as a pathoetiological stressor to trigger the subsequent development of FM. ${ }^{23}$ Physical traumas, including traffic accidents, may thus act as a trigger for FM. This finding provides an important basis for further understanding the pathogenesis of FM and requires further investigation. In addition, when treating patients after a traffic accident, physicians might consider FM if treatments are ineffective or if patient's pain is unexpectedly prolonged.

While it offers significant benefits to the clinical and FM literature, this study has some limitations. First, we did not investigate the ultimate disease diagnosis and the treatment of participants enrolled in our study who had suspected FM. Because the diagnosis of FM is made when other diseases are excluded, the prevalence rate can be lowered if another diagnosis is made. However, as mentioned earlier, if FM is considered to be a continuum disorder rather than a single disease, our study suggests that many patients with chronic pain have the FM phenotype. Second, not all patients seen at all study sites during the study period were included in this study. If all patients had been included, the prevalence of FM reported here might have differed. Finally, an official Korean version of the modified 2010 ACR criteria has not yet been published. Future studies of FM in Korea would thus first require validation of a translated version, such as that used here.

\section{Conclusion}

The present study demonstrates a high prevalence of FM, according to the modified 2010 ACR criteria, among chronic pain patients referred to tertiary care university hospital pain clinics in Korea. Because FM is difficult to diagnose in the absence of clinical suspicion, pain physicians should consider the elevated prevalence of FM in patients who visit their clinics. In addition, the modified 2010 ACR criteria will be useful because they can be applied easily without the intervention of a physician. Despite some limitations, the present study provides a meaningful contribution to the literature as the first multicenter epidemiological study of FM in Korea. It is also the first study to use the modified 2010 ACR criteria in Korea. The results of this study are expected to be utilized in future epidemiological research on FM in Korea.

\section{Acknowledgments}

The authors thank the Division of Statistics in the Medical Research Collaborating Center at Seoul National University Bundang Hospital for assistance with the statistical analyses. The authors thank the following people for collecting the data at each hospital. Dae Hyun Jo, Department of Anesthesiology and Pain Medicine, Daejeon St. Mary's Hospital, Daejeon, Korea; Sang Sik Choi, Department of Anesthesiology and Pain Medicine, Korea University Guro Hospital, Seoul, Korea; Dae Hyun Kim, Department of Anesthesiology and Pain Medicine, National Cancer Center, Goyang, Korea; Woo Seok Shim, Department of Anesthesiology and Pain Medicine, Samsung Medical Center, Seoul, Korea; Yeon Dong Kim, Department of Anesthesiology and Pain Medicine, Wonkwang University Hospital, School of Medicine, Wonkwang University, Wonkwang Institute of Science, Iksan, Korea; Jeong Il Choi, Department of Anesthesiology and Pain Medicine, Medical School, Chonnam National University, Gwangju, Korea; Yun Suk Choi, Department of 
Anesthesiology and Pain Medicine, Jeju National University School of Medicine, Jeju, Korea; Sun Yeul Lee, Department of Anesthesiology and Pain Medicine, College of Medicine, Chungnam National University, Daejeon, Korea; and Ji-Seok Baek, Department of Anesthesiology and Pain Medicine, Pusan National University, Pusan, Korea. This research did not receive any specific grant from funding agencies in the public, commercial, or not-for-profit sectors.

\section{Disclosure}

The authors report no conflicts of interest in this work.

\section{References}

1. McMahon SB, Koltzenburg M, Tracey I, Turk D. Wall \& Melzack's Textbook of Pain E-Book. 6th ed. Philadelphia: Elsevier; 2013.

2. Häuser W, Ablin J, Fitzcharles MA, et al. Fibromyalgia. Nat Rev Dis Primers. 2015;1:15022.

3. Wolfe F, Clauw DJ, Fitzcharles MA, et al. The American College of Rheumatology preliminary diagnostic criteria for fibromyalgia and measurement of symptom severity. Arthritis Care Res (Hoboken). 2010;62(5):600-610.

4. Wolfe F, Clauw DJ, Fitzcharles MA, et al. Fibromyalgia criteria and severity scales for clinical and epidemiological studies: a modification of the ACR Preliminary Diagnostic Criteria for Fibromyalgia. J Rheumatol. 2011;38(6):1113-1122.

5. Ferrari R, Russell AS. A questionnaire using the modified 2010 American College of Rheumatology criteria for fibromyalgia: specificity and sensitivity in clinical practice. J Rheumatol. 2013;40(9):1590-1595.

6. Wolfe F, Brähler E, Hinz A, Häuser W. Fibromyalgia prevalence, somatic symptom reporting, and the dimensionality of polysymptomatic distress: results from a survey of the general population. Arthritis Care Res (Hoboken). 2013;65(5):777-785.

7. Kim SH, Bae GR, Lim HS. Prevalence and risk factors of fibromyalgia syndrome and chronic widespread pain in two communities in Koreafirst report in Korean. J Korean Rheum Assoc. 2006;13(1):18-25.

8. Kim C, Kim H, Kim J. Prevalence of chronic widespread pain and fibromyalgia syndrome: a Korean hospital-based study. Rheumatol Int. 2012;32(11):3435-3442.

9. Kim SM, Lee SH, Kim HR. Applying the ACR preliminary diagnostic criteria in the diagnosis and assessment of fibromyalgia. Korean J Pain. 2012;25(3):173-182.
10. Giesecke T, Gracely RH, Grant MA, et al. Evidence of augmented central pain processing in idiopathic chronic low back pain. Arthritis Rheum. 2004;50(2):613-623.

11. Glaziu P. Sampsize calculator [webpage on the Internet]. 2003 [updated July 30, 2005]. Available from: http://sampsize.sourceforge.net/iface/ index.html. Accessed May 8, 2016

12. Wolfe F, Smythe HA, Yunus MB, et al. The American College of Rheumatology 1990 criteria for the classification of fibromyalgia. Report of the Multicenter Criteria Committee. Arthritis Rheum. 1990;33(2):160-172.

13. Jones GT, Atzeni F, Beasley M, Flüß E, Sarzi-Puttini P, Macfarlane GJ. The prevalence of fibromyalgia in the general population: a comparison of the American College of Rheumatology 1990, 2010, and modified 2010 classification criteria. Arthritis Rheumatol. 2015;67(2): $568-575$.

14. Vincent A, Lahr BD, Wolfe F, et al. Prevalence of fibromyalgia: a population-based study in Olmsted County, Minnesota, utilizing the Rochester Epidemiology Project. Arthritis Care Res (Hoboken). 2013;65(5):786-792.

15. Nakamura I, Nishioka K, Usui C, et al. An epidemiologic internet survey of fibromyalgia and chronic pain in Japan. Arthritis Care Res (Hoboken). 2014;66(7):1093-1101.

16. Weir PT, Harlan GA, Nkoy FL, et al. The incidence of fibromyalgia and its associated comorbidities: a population-based retrospective cohort study based on International Classification of Diseases, 9th Revision codes. J Clin Rheumatol. 2006;12(3):124-128.

17. Hughes G, Martinez C, Myon E, Taïeb C, Wessely S. The impact of a diagnosis of fibromyalgia on health care resource use by primary care patients in the UK: an observational study based on clinical practice. Arthritis Rheum. 2006;54(1):177-183.

18. Choy E, Perrot S, Leon T, et al. A patient survey of the impact of fibromyalgia and the journey to diagnosis. BMC Health Serv Res. 2010;10:102.

19. Brill S, Ablin JN, Goor-Aryeh I, et al. Prevalence of fibromyalgia syndrome in patients referred to a tertiary pain clinic. J Investig Med. 2012;60(4):685-688.

20. Yunus MB. The role of gender in fibromyalgia syndrome. Curr Rheumatol Rep. 2001;3(2):128-134.

21. Bigatti SM, Hernandez AM, Cronan TA, Rand KL. Sleep disturbances in fibromyalgia syndrome: relationship to pain and depression. Arthritis Rheum. 2008;59(7):961-967.

22. Leavitt F, Katz RS, Mills M, Heard AR. Cognitive and dissociative manifestations in fibromyalgia. J Clin Rheumatol. 2002;8(2):77-84.

23. McLean SA, Williams DA, Clauw DJ. Fibromyalgia after motor vehicle collision: evidence and implications. Traffic Inj Prev. 2005;6(2): 97-104.
Journal of Pain Research

\section{Publish your work in this journal}

The Journal of Pain Research is an international, peer reviewed, open access, online journal that welcomes laboratory and clinical findings in the fields of pain research and the prevention and management of pain. Original research, reviews, symposium reports, hypothesis formation and commentaries are all considered for publication.

\section{Dovepress}

The manuscript management system is completely online and includes a very quick and fair peer-review system, which is all easy to use. Visit http://www.dovepress.com/testimonials.php to read real quotes from published authors. 